UCT-TP-209/94

March 1994

\title{
Operator product expansion and duality at finite temperature
}

\author{
C. A. Dominguezt \\ Institute of Theoretical Physics and Astrophysics, University of Cape Town, Rondebosch 7700, \\ South Africa
}

\begin{abstract}
The operator product expansion of current correlators at short distances, and the notion of QCD-hadron duality are the cornerstone of QCD sum rules. The extension of this programme to $T \neq 0$ is discussed, together with applications to hot hadronic propagators. Indications are that the hadronic spectrum suffers a substantial rearrangement with increasing temperature, and hint on the existence of a quark deconfining phase transition. Phenomenological order parameters to characterize this phase transition are discussed.
\end{abstract}

Some time ago Bochkarev and Shaposnikov [1] proposed an extension of the QCD sum rule program to non-zero temperature, and made an application to the two-point function involving the vector current. This application was reconsidered in [2]. Later on we discussed the axialvector channel [3] and the nucleon channel 酒 using Finite Energy QCD sum rules (FESR). Results from these analyses indicate a substantial rearrangement of the hadronic spectrum with increasing temperature, and hint on the existence of a deconfining phase transition. This was later confirmed in [5], where a formalism valid even for $\mathrm{T}$ near the critical temperature was used. An extension of QCD sum rules to finite temperature entails the assumptions that (a) the OPE continues to be valid, except that now the vacuum condensates will develop an (a-priori) unknown temperature dependence, and (b) the notion of QCD-hadron duality also remains valid. At $\mathrm{T}=0$, the thermal behaviour of the vacuum condensates is not calculable analytically from first principles; some model or approximation must be invoked, e.g. the dilute pion gas approximation, lattice QCD, etc.. At $T=0$, QCD-hadron duality is well established phenomenologically, while the validity of the OPE in QCD (in the presence of non-perturbative phenomena) is made plausible after studying other field theories which can be solved exactly [6]. At $T \neq 0$, it is important to examine critically the basis for the two assumptions above, especially in the light of some recent adverse criticisms [7].

The basic object under consideration is the retarded (advanced) two-point function, involving a local current operator $J(x)$, after appropriate Gibbs averaging

$$
\Pi(q, T)=i \int d^{4} x \exp (i q x) \theta\left(x_{0}\right)<<\left[J(x), J^{\dagger}(0)\right]>>,
$$

\footnotetext{
${ }^{1}$ Invited talk at QCD-94, Montpellier, July 1994

${ }^{2}$ John Simon Guggenheim Fellow 1994-1995
} 
where

$$
<<A \cdot B>>=\sum_{n} \exp \left(-E_{n} / T\right)\langle n|A \cdot B| n\rangle / \operatorname{Tr}(\exp (-H / T))
$$

and $|n\rangle$ is a complete set of eigenstates of the (QCD) Hamiltonian. The $\operatorname{OPE}$ of $\Pi(q, T)$ is formally written as

$$
\Pi(q, T)=C_{I}<<I>>+\sum_{r} C_{r}(q)<<\mathcal{O}_{r}>>,
$$

The unit operator $I$ in Eq.(3) represents the purely perturbative piece, and the OPE is assumed valid, even in the presence of non-perturbative effects, for $q^{2}<0$ (spacelike), and $\left|q^{2}\right| \gg \Lambda_{Q C D}^{2}$. The states $\mid n>$ entering Eq.(2) can be any complete set of states, e.g. hadronic states, quarkgluon basis, etc.. If one wishes to extend smoothly the $T=0$ QCD sum rule program to finite temperature, then the natural choice for this set is the quark-gluon basis, as first proposed in [1]. I discuss below some arguments in support of the validity of the OPE at $T \neq 0$, Eq.(3). Assuming this, and invoking duality at $T=0$, one now raises the temperature by an arbitrary small value. The hadronic spectrum and the expectation values of the QCD operators in the OPE will hardly change. Hence, it is reasonable to expect that the inter-relationship between QCD and hadronic parameters effected by duality will remain valid. An abrupt dissapearance of this inter-relationship as soon as $T \neq 0$, as advocated in [7], appears as an unlikely possibility. One should keep in mind that the current in Eq.(1) is an object external to the heat bath, and thus need not be in thermal equilibrium with the particles in the medium. The fact that at low $T$ these particles are predominantly hadrons, is irrelevant for the argument. The external current will still convert to quark-antiquark pairs, which contribute e.g. to the perturbative operator $I$ through loops, where they can have any value of momentum. In addition, there will be thermal non-perturbative effects as parametrized by the condensates. These are always distinguishable from perturbative effects, since they have a different $q^{2}$ dependence. In many cases they have also a different T-dependence, and may contribute only to the real part of the correlators. As the temperature is increased further, the hadronic spectrum will begin to change shape, in pace with the thermal behaviour of the expectation values of the various operators in the OPE. I shall discuss later how these changes emerge from QCD sum rules in agreement with expectations from general physical considerations.

I turn now to the validity of the OPE at $T \neq 0$. Just as at $T=0$, no rigorous proof can be given since one cannot solve QCD analytically and exactly. Instead, other field theory models which can be solved exactly have been used to argue for the validity of the OPE at $T=0$ [6]. These models include the $O(N)$ sigma model in the large $N$ limit, and the Schwinger model, both in two dimensions. Comparing the short distance expansion of the exact solution for a Green function with the OPE counterpart, one finds that they are identical [6]. One can also show that this is also the case at finite temperature [8], as I outline next. Let us consider first the $\mathrm{O}(\mathrm{N})$ sigma model in $1+1$ dimensions which is characterized by the Lagrangian

$$
\mathcal{L}=\frac{1}{2}\left[\partial_{\mu} \sigma^{a}(x)\right]\left[\partial_{\mu} \sigma^{a}(x)\right],
$$

where $\mathrm{a}=1, \ldots \mathrm{N}$ and $\sigma^{a} \sigma^{a}=N / f$, with $\mathrm{f}$ being the coupling constant. In the large $\mathrm{N}$ limit this model can be solved exactly (for details see [6]), it is known to be asymptotically free, and in spite of the absence of mass parameters in Eq.(4), it exhibits dynamical mass generation. In 
addition, in this model there are vacuum condensates, e.g. to leading order in $1 / \mathrm{N}:\langle 0|\alpha| 0\rangle=$ $\sqrt{N} m^{2}$, whereas all other condensates factorize, viz. $\left\langle 0\left|\alpha^{k}\right| 0\right\rangle=\left(\sqrt{N} m^{2}\right)^{k}$. The $\alpha$ field is: $\alpha=f\left(\partial_{\mu} \sigma^{a}\right)^{2} / \sqrt{N}$, and we are interested in the Green function associated with the propagation of quanta of this $\alpha$ field. We have calculated this Green function at finite temperature. Its imaginary part can be integrated analytically in closed form and is

$$
\begin{aligned}
\operatorname{Im} \Gamma(\omega, \mathbf{q}=0, T) & =\frac{1}{2 \omega^{2}}\left[1+3 n_{B}(\omega / 2 T)\right] \\
& +\frac{1}{2}\left[\frac{2}{\sqrt{N}} \frac{\langle<\alpha>>}{\omega^{4}}+\frac{6}{N} \frac{\left\langle<\alpha^{2}\right\rangle>}{\omega^{6}}+\cdots\right],
\end{aligned}
$$

where the first term above corresponds to the perturbative contribution, the second to the nonperturbative, and $n_{B}$ is the thermal Bose factor. Equation (5) is valid in the time-like region; the space-like region counterpart vanishes in 2 dimensions. Since the model is exactly solvable, the thermal behaviour of the vacuum condensates can also be calculated, viz.

$$
<<\alpha>>=<\alpha>\left[1+3 n_{B}(\omega / 2 T)\right]
$$

In this case the vacuum condensates contribute to the imaginary part, and as Eq.(5) shows, the thermal dependence of the perturbative piece cannot be absorbed into the condensates. Hence, no confusion should arise between perturbative and non-perturbative contributions.

Finally, I consider the Schwinger model in $1+1$ dimensions, with the Lagrangian

$$
\mathcal{L}=-\frac{1}{4} F_{\mu \nu} F_{\mu \nu}+\bar{\psi} i \gamma_{\mu} \mathcal{D}_{\mu} \psi
$$

where $\mathcal{D}_{\mu}=i \partial_{\mu}+e A_{\mu}$. This model has been solved exactly, and in the framework of the OPE [6]. The short distance expansion of the exact solution coincides with that from the OPE [6]. Here, we are interested in the two-point functions

$$
\begin{aligned}
& \Pi_{++}(x)=\left\langle 0\left|T\left\{j^{+}(x) j^{+}(0)\right\}\right| 0\right\rangle \\
& \Pi_{+-}(x)=\left\langle 0\left|T\left\{j^{+}(x) j^{-}(0)\right\}\right| 0\right\rangle
\end{aligned}
$$

where the scalar currents are: $j^{+}=\bar{\psi}_{R} \psi_{L}, j^{-}=\bar{\psi}_{L} \psi_{R}$, with $\psi_{L, R}=\left(1 \pm \gamma_{5}\right) \psi / 2$. The function $\Pi_{++}(Q)$ vanishes identically in perturbation theory, and the leading non-perturbative contribution involves a four-fermion vacuum condensate. We have calculated the thermal behaviour of these current correlators and obtain, e.g. for their imaginary parts in the time-like region (again, there is no space-like contribution in 2 dimensions)

$$
\begin{gathered}
\operatorname{Im} \Pi_{++}(\omega, \mathbf{q}=0, T)=0 \\
\operatorname{Im} \Pi_{+-}(\omega, \mathbf{q}=0, T)=\frac{1}{4}\left[1-2 n_{F}(\omega / 2 T)\right]
\end{gathered}
$$

Hence, the choice of the fermion basis in the Gibbs average of current correlators does not imply confusing these fermions with condensates, as argued in [7]. As Eqs.(10) and (11) indicate, (perturbative) fermion loop terms and (non-perturbative) vacuum condensates develop their own temperature dependence, which in this particular example happen to be different. 
Turning to applications, an inspection of a typical hadronic spectral function at $T=0$ shows some resonance peaks at low energy, followed by a hadronic continuum starting at some threshold $s_{0}$. Because of asymptotic freedom, this continuum is well approximated by perturbative QCD. Raising the temperature, one would expect resonance melting. This may be accomplished by the imaginary part of hadronic propagators growing with temperature, as first proposed in [9]. As the resonance peaks become broader, one would expect $s_{0}$ to decrease. Close to the critical temperature for deconfinement, the hadronic spectral function should be rather smooth, and represented almost entirely by the quark-gluon degrees of freedom over the whole energy range. An indication for some decrease of $s_{0}$ with increasing $T$ was obtained from QCD sum rules for the vector channel [1] - 22], [10]. However, it is the axial channel the one that provides conclusive evidence for this behaviour [3], [5]. In fact, the solution to the FESR gives

$$
\frac{s_{0}(T)}{s_{0}(0)} \simeq \frac{f_{\pi}^{2}(T)}{f_{\pi}^{2}(0)}
$$

The temperature behaviour of the imaginary part of a hadronic propagator is difficult to obtain from QCD sum rules, since these are not too sensitive to resonance widths. Independent calculations in other theoretical frameworks [11] show that the pion, the nucleon, and the rho-meson propagators have imaginary parts which grow monotonically with $T$. Over a certain range of temperatures these behave as

$$
\Gamma(T)=\frac{\Gamma(0)}{\left(1-T / T_{c}\right)^{\alpha}} .
$$

Thus, the asymptotic freedom threshold $s_{0}(T)$, and the resonance width $\Gamma(T)$, are suitable phenomenological order parameters to characterize the quark deconfinement phase transition. It should be mentioned, in closing, that there are some unresolved problems in finite temperature QCD sum rules, e.g. when one tries to use them to extract the thermal behaviour of the vacuum condensates [10]. An unwelcome implication is a breakdown of the FESR program beyond the lowest moment sum rule, and hence a breakdown of Laplace transform QCD sum rules. A satisfactory resolution of this problem is eagerly awaited.

\section{Acknowledgements}

This work was done in collaboration with M. Loewe. The author wishes to thank Stephan Narison for a perfectly organized, and most enjoyable workshop.

\section{References}

[1] A.I.Bochkarev and M.E.Shaposnikov, Nucl. Phys.B 268 (1986) 220.

[2] H.G.Dosch and S.Narison, Phys. Lett. B 203 (1988) 155.

[3] C.A.Dominguez and M.Loewe, Phys. Lett. B 233 (1989) 201.

[4] C.A.Dominguez and M.Loewe, Z. Phys. C - Particles and Fields - 58 (1993) 273. 
[5] A.Barducci et al., Phys. Lett. B 244 (1990) 311.

[6] V.A.Novikov, M.A.Shifman, A.I.Vainshtein and V.I.Zakharov, Phys. Rep. C 116 (1984) 103; Nucl. Phys. B 249 (1985) 445.

[7] M. Dey, V.L.Eletsky and B.L.Ioffe, Phys. Lett. B 252 (1990) 620 ; V.L.Eletsky and B.L.Ioffe, Phys. Rev. D 47 (1993) 3083.

[8] C.A.Dominguez and M.Loewe, UCT Report No. UCT-TP-208/94.

[9] C.A.Dominguez and M.Loewe, Nucl. Phys. B (Proc. Suppl.)16 (1990) 403 .

[10] C.A.Dominguez and M.Loewe, Z. Phys. C - Particles and Fields - 51 (1991) 69.

[11] J.Goity and H.Leutwyler, Phys. Lett. B 228 (1989) 517 ; H.Leutwyler and V.Smilga, Nucl. Phys. B 342 (1990) 302; A.Schenk, Nucl. Phys. B 363 (1991) 97; C.A.Dominguez, M.Loewe, and J.C.Rojas, Phys. Lett. B 320 (1994) 377. 